\title{
Circular RNA F-circEA-2a derived from EML4-ALK fusion gene promotes cell migration and invasion in non-small cell lung cancer
}

Shuangyan Tan ${ }^{\dagger}$, Dan Sun ${ }^{\dagger}$, Wenchen Pu, Qiheng Gou, Chenglin Guo, Youling Gong, Jiao Li, Yu-Quan Wei, Lunxu Liu*, Yun Zhao ${ }^{*}$ and Yong Peng* (i)

\begin{abstract}
Oncogenic fusion gene Echinoderm Microtubule-associated protein-Like 4-Anaplastic Lymphoma Kinase (EML4-ALK) contributes to tumorigenesis of a subset of non-small cell lung cancer (NSCLC). Recently, we demonstrated that F-circEA-4a, a tumor-promoting circular RNA (circRNA) generated from the back-splicing of EML4-ALK variant 3b ( $\mathrm{v} 3 \mathrm{~b})$, is a novel liquid biopsy biomarker for NSCLC. However, circRNAs produced from EML4-ALK gene and their roles in NSCLC are not well-characterized. Here, we identify another EML4-ALK-v3b-derived circRNA, F-circEA-2a, harboring "AA" (rather than "AAAA" in F-circEA-4a) motif at the junction site. F-circEA-2a mainly locates in the cytoplasm and promotes cell migration and invasion, but has little effect on cell proliferation. Moreover, F-circEA-2a exists in tumor, but not in the plasma of NSCLC patients with EML4-ALK fusion gene, further supporting the significant diagnostic value of F-circEA-4a for EML4-ALK-positive NSCLC. This work finds a novel oncogenic circRNA generated from EML4-ALK fusion gene, highlighting the pivotal role of circRNA in EML4-ALK-positive NSCLC development.
\end{abstract}

Keywords: Non-small cell lung cancer, EML4-ALK, Circular RNA, Cell migration/invasion

\section{Main text}

Lung cancer is the leading cause of cancer death worldwide, in which non-small cell lung cancer (NSCLC) is a main subgroup accounting for approximately $85 \%$ of all lung cancer cases [1]. NSCLC patients are often diagnosed at advanced stage and their 5-year survival rate is extremely low [2]. Thus, investigation of NSCLC-associated process is urgent for NSCLC diagnosis and treatment.

A subset of NSCLC harbor fusion gene which encodes fusion protein to exert oncogenic phenotype. For example, Echinoderm Microtubule-associated protein-Like 4-Anaplastic Lymphoma Kinase (EML4-ALK) fusion gene is present in $4-5 \%$ of NSCLC cases and generates $E M L 4-A L K$ fusion protein to activate ALK-associated

\footnotetext{
* Correspondence: lunxu_liu@aliyun.com; zhaoyun@scu.edu.cn; yongpeng@scu.edu.cn

†Shuangyan Tan and Dan Sun contributed equally to this work. Key Laboratory of Bio-Resource and Eco-Environment of Ministry of Education, College of Life Sciences; Department of Thoracic Surgery, State Key Laboratory of Biotherapy, West China Hospital, Sichuan University, Chengdu 610041, China
}

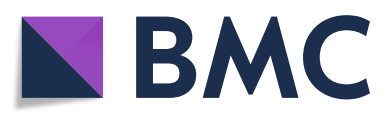

(c) The Author(s). 2018 Open Access This article is distributed under the terms of the Creative Commons Attribution 4.0 International License (http://creativecommons.org/licenses/by/4.0/), which permits unrestricted use, distribution, and reproduction in any medium, provided you give appropriate credit to the original author(s) and the source, provide a link to the Creative Commons license, and indicate if changes were made. The Creative Commons Public Domain Dedication waiver (http://creativecommons.org/publicdomain/zero/1.0/) applies to the data made available in this article, unless otherwise stated.

oncogenic signaling and promote NSCLC progression [3-5]. However, their underlying mechanism in NSCLC remains obscure.

Circular RNAs (circRNAs) are a special subtype of non-coding RNAs with circular covalently-bonded structure, which endows them higher tolerance to exonucleases. Due to their conservation, abundance and specificity, circRNAs participate in diverse physiological and pathological processes, including tumorigeneisis [6]. Increasing evidence demonstrate that circRNAs derived from the back-splicing of fusion gene are alternative entities involved in cancer development besides fusion proteins. For instance, the circRNA generated by $M L L / A F 9$ fusion gene (f-circM9) in leukemia shows pro-proliferative and pro-oncogenic activities [7]. Additionally, we recently demonstrated that fusion gene $E M L 4-A L K$ variant $3 \mathrm{~b}$ (v3b) produces an oncogenic circRNA (F-circEA-4a) with "AAAA" motif at the junction site. Importantly, F-circEA-4a could be detected in the plasma of EML4$A L K$-positive NSCLC patients, acting as a potential liquid 
biopsy biomarker [8]. However, circRNA-producing potential of EML4-ALK gene and the role of these circRNAs in NSCLC are not fully understood.

Here, we identify another circRNA F-circEA-2a produced from $E M L 4-A L K$-v3b with "AA" motif at the junction site. Moreover, F-circEA-2a has little effect on cell proliferation, but promotes cell migration and invasion in NSCLC cells, highlighting the critical role of circRNAs in EML4-ALK-positive NSCLC.

\section{Results and discussion}

\section{Identification of F-circEA-2a in NSCLC}

We recently identified the existence of EML4-ALK-derived circRNA F-circEA-4a. To evaluate whether EML4-ALK fusion gene could produce other circRNAs, we deeply Sanger-sequenced the reverse transcription PCR (RT-PCR) products using divergent F1/R1 primers (Fig. 1a) from H2228 (harboring EML4-ALK-v3b) and A549 cells (without the fusion gene, negative control). Besides F-circEA-4a, we found the presence of another circRNA named as F-circEA-2a with "AA" motif at the junction site in the RNA sample from H2228 cells, which was treated with RNase R to remove linear RNAs
(Fig. 1b). F-circEA-2a, about $0.55 \mathrm{~kb}$ in length, is the back-splicing product between $5^{\prime}$-head of EML4 exon4 and 3'-tail of ALK exon22 (Fig. 1a). Dot blot hybridization using ${ }^{32} \mathrm{P}$-labeled probes across the respective junction sites indicated that the enrichment of F-circEA-2a in H2228 cells was less than that of F-circEA-4a (Fig. 1c, left), which was further confirmed by quantitative PCR (qPCR) (Fig. 1c, right). Subcellular fractionation and qPCR assays showed that F-circEA-2a was mainly located in the cytoplasm (Fig. 1d). These data demonstrated the presence of another circRNA F-circEA-2a produced from EML4-ALK fusion gene.

\section{F-circEA-2a promotes cell migration and invasion in NSCLC cells}

To investigate cellular function of F-circEA-2a, we constructed F-circEA-2a-expressing plasmid, in which F-circEA-2a sequences (red arrows) and the flanking sequences (green arrows, favorable for circRNA formation) were cloned into pCDH-CMV-MCS-EF1-puro vector (Fig. 2a). Sanger sequencing of RT-PCR products from the cells transfected with F-circEA-2a-expressing plasmid indicated that F-circEA-2a was successfully

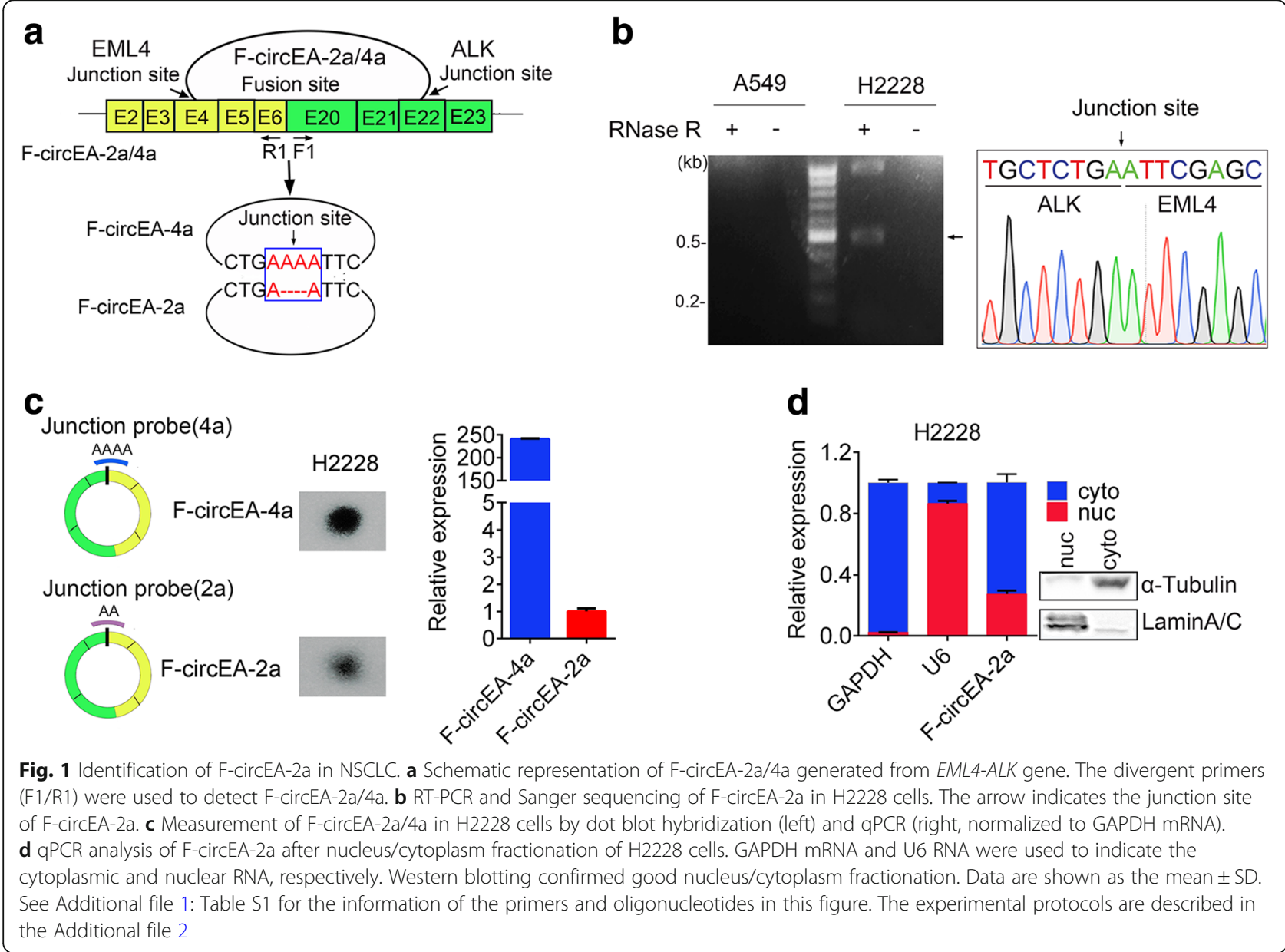


a

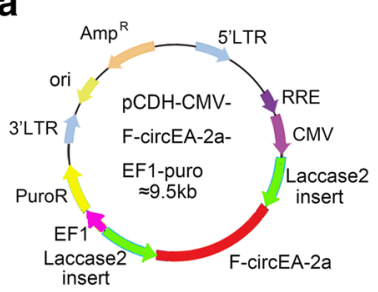

e

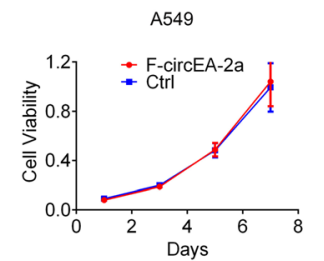

g
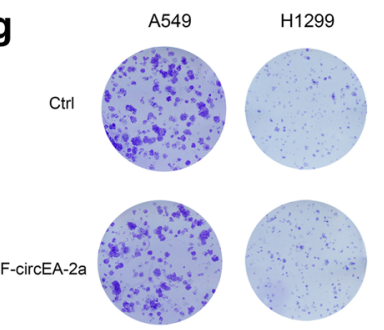

C

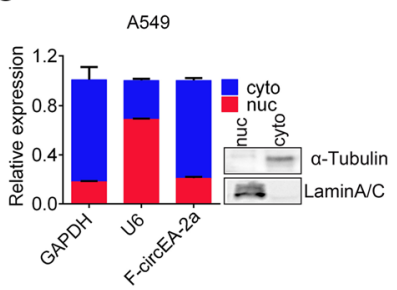

d

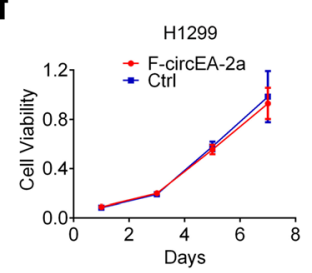

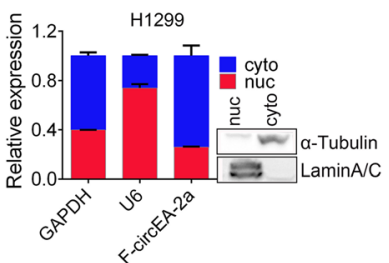

$\mathbf{h}$

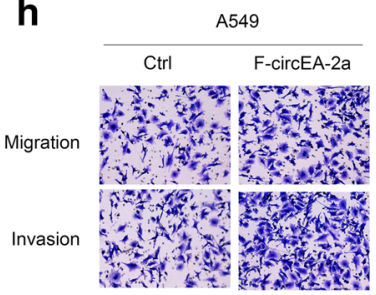

i
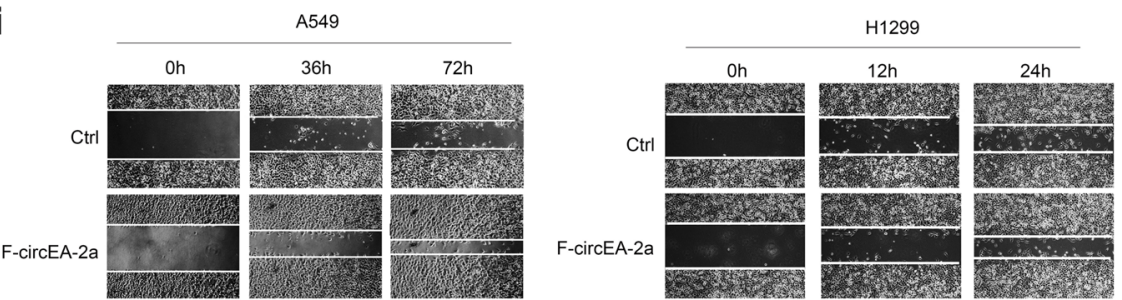

Fig. 2 F-circEA-2a promotes cell migration and invasion in NSCLC cells. a Schematic representation of F-circEA-2a-expressing plasmid with the flanking sequence of laccase 2 to facilitate RNA circularization. b Agarose gel electrophoresis and Sanger sequencing of RT-PCR products from H1299 cells transfected with F-circEA-2a-expressing plasmid and empty vector. c.d Nucleus/cytoplasm fractionation and qPCR analysis of F-circEA-2a in A549 (c) and H1299 (d) cells. Western blotting against laminA/C and tubulin showed efficient nucleus/cytoplasm fractionation. Data are shown as the mean \pm SD. e.f.g MTT (e.f) and colony formation assays (g) in A549 and H1299 cells transfected with F-circEA-2a-expressing plasmid or empty vector (Ctrl). h.i Representative images of Transwell (h) and wound-healing assays (i) in A549 and H1299 cells transfected with F-circEA-2a-expressing plasmid or empty vector (Ctrl). See Additional file 1:Table S1 for the information of the primers and oligonucleotides in $\mathbf{a}$ and $\mathbf{b}$. The experimental protocols are described in the Additional file 2

expressed and correctly back-spliced (Fig. 2b). The ectopically expressed F-circEA-2a was predominantly located in the cytoplasm of both H1299 and A549 cells (Fig. 2c and d), same as the endogenous one in H2228 cells.

Both MTT and colony formation assays suggested that ectopically expressed F-circEA-2a had no significant effect on cell proliferation in A549 and H1299 cells (Fig. 2e, f and g). However, F-circEA-2a enhanced cell migration and invasion in both cells by Transwell assays and wound-healing experiments (Fig. $2 \mathrm{~h}$ and i). Because both F-circEA-4a and F-circEA-2a can promote cell migration and their difference is that F-circEA-4a has extra
AA dinucleotides at the junction site, so they may exert the cellular function through the same mechanism. Emerging evidence shows that circRNAs play an important role under physiological or pathological conditions. For example, circMTO1, down-regulated circular RNA in hepatocellular carcinoma (HCC), suppresses HCC progression by acting as the sponge of oncogenic miR-9 to promote p21 expression [9]. These data reveal a novel circRNA F-circEA-2a enhances cell migration and invasion without any influence on cell proliferation, enlarging the understanding of circRNA as oncogenic molecule participating tumor development. 

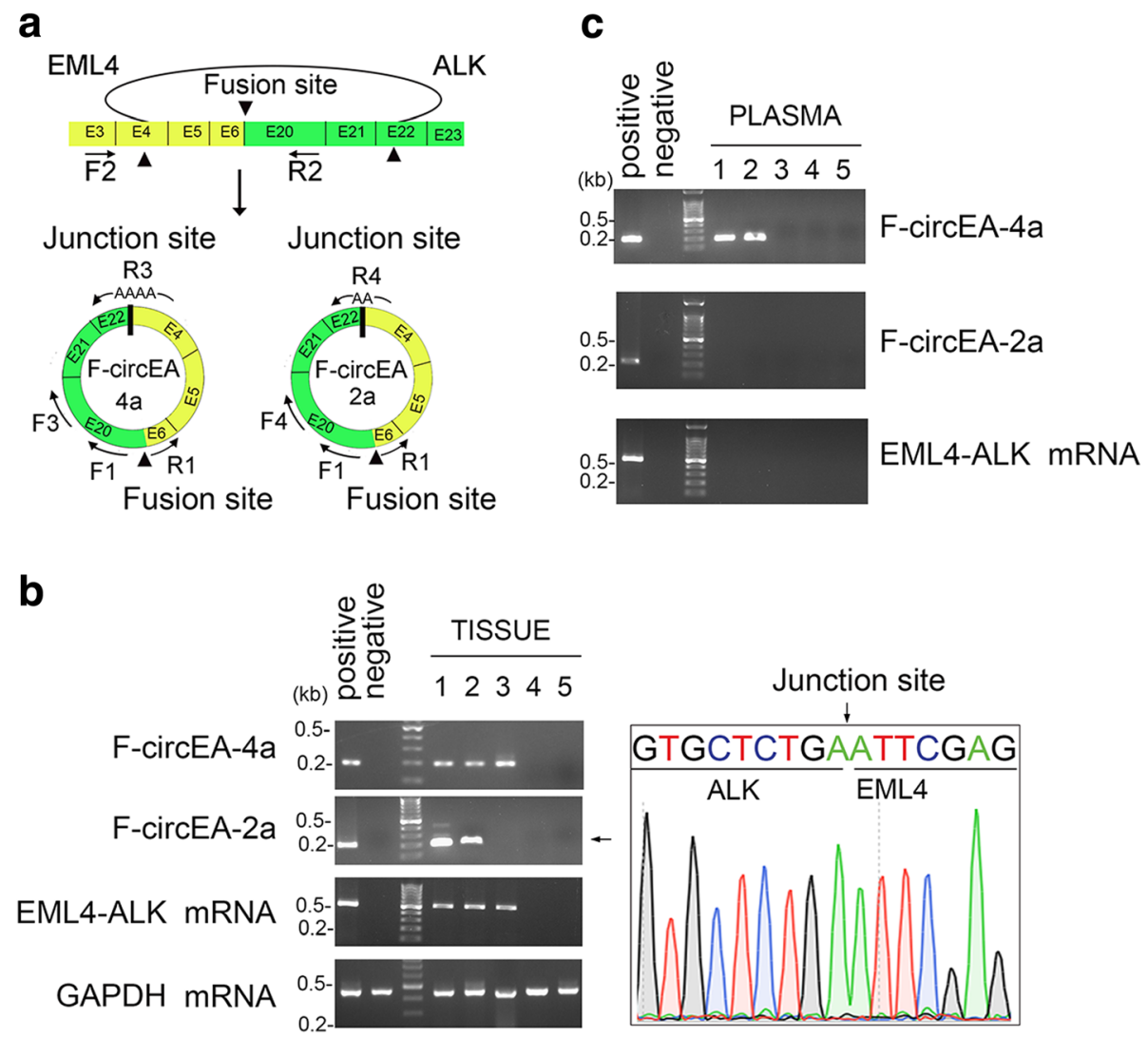

Fig. 3 Identification of F-circEA-2a in NSCLC patients' samples. a Primers used to detect EML4-ALK mRNA and F-circEA-2a/4a. The convergent primers (F2/R2) were used to detect EML4-ALK mRNA, the divergent primers F3/R3 and F4/R4 were used to detect F-circEA-4a/2a, respectively. b.c Agarose gel electrophoresis and Sanger sequencing of RT-PCR products from tumor tissues (b) or plasma (c) of NSCLC patients with (patients 1-3) or without (patients 4-5) EML4-ALK variant 3b translocation. See Additional file 1:Table S1 for the information of the primers and oligonucleotides in this figure. The experimental protocols are described in the Additional file 2

\section{Detection of F-circEA-2a in NSCLC patient}

Compared to linear RNAs, the covalently-bonded circRNAs are relatively resistance to RNase $\mathrm{R}$ digestion. Some circRNAs (such as circ-N4BP2L2, circ-GSE1) originated from cancer tissues may enter the circulating system and stably be present in intercellular fluid [10]. Our recent work indicates that F-circEA-4a is a potential liquid biopsy biomarker for EML4-ALK-positive NSCLC patients [8]. To investigate the clinical significance of F-circEA-2a, we measured F-circEA-2a levels in tumor tissues and plasma of three NSCLC patients with EML4-ALK-v3b translocation and two NSCLC patients without such fusion gene. The convergent primers (F2/R2) were also used to detect EML4-ALK fusion mRNA. To improve specificity, we designed nested divergent primers (Fig. 3a, first round PCR primers: F1/R1; nested PCR primers for F-circEA-4a: F3/R3, nested PCR primers for F-circEA-2a: F4/R4). The R3 and R4 primers were designed to cross the junction sites of F-circEA-4a and F-circEA-2a, respectively, facilitating specific detection of these
circRNAs (Fig. 3a). RT-PCR and Sanger sequencing data demonstrated that F-circEA-4a, F-circEA-2a and $E M L 4-A L K$ mRNA were all detected in the tumor tissues of NSCLC patients with EML4-ALK-v3b translocation, whereas they were absent in the patients without such fusion gene (Fig. 3b), indicating the specific existence of F-circEA-4a and F-circEA-2a in the EML4-ALK-positive NSCLC tumors. However, in contrast to F-circEA-4a, we failed to detect F-circEA-2a in the plasma of EML4-ALK-positive NSCLC patients (Fig. 3c), which may be caused by low enrichment or a discrepant junction motif of F-circEA-2a. These results provided a rebuttal that F-circEA-4a is a promising biomarker for $E M L 4-A L K$-v3b translocation, highlighting its uncontroversial role in the liquid biopsy of EML4-ALK-positive NSCLC patients.

In summary, a novel circRNA F-circEA-2a produced from the EML4-ALK fusion gene was identified and mainly located in the cytoplasm to promote cell migration and invasion in lung cancer cells. 


\section{Additional files}

Additional file 1: Table S1. Information of primers and oligonucleotides used in this study. (DOCX $16 \mathrm{~kb})$

Additional file 2: Experimental materials and methods in this study. (DOCX $92 \mathrm{~kb}$ )

\section{Abbreviations}

circRNAs: Circular RNAs;"; EML4-ALK: Echinoderm Microtubule-associated protein-Like 4-Anaplastic Lymphoma Kinase:" NSCLC: Non-small cell lung cancer; qPCR: Real-time quantitative polymerase chain reaction; RT-PCR: Reverse transcription polymerase chain reaction

\section{Acknowledgements}

We'd like to thank Prof. Jeremy E. Wilusz for providing the plasmid pcDNA3.1(+) Laccase2 MCS Exon, and all of our laboratory members for discussions.

\section{Funding}

This work was supported by the National Key R\&D Program of China (2017YFA0504304 and 2016YFA0502204 to Y.P.), National Natural Science Foundation of China (81772960 and 81572739 to Y.P.), and Graduate Student's Research and Innovation Fund of Sichuan University (2018YJSY017 to D.S.).

\section{Availability of data and materials}

All data obtained and/or analyzed during the current study were available from the corresponding authors in a reasonable request.

\section{Authors' contributions}

YP, YZ and YW conceived and designed the project; ST, DS, WP, QG and JL performed the experiments and interpreted data; CG, YG and LL collected NSCLC patients' samples; all authors read and approved the final manuscript.

\section{Ethics approval and consent to participate}

The human cancer tissues and plasma used in this study were approved by the Ethics Committee of West China Hospital of Sichuan University.

\section{Consent for publication}

We have received consents from individual patients who participated in this study. The consent forms will be provided upon request.

\section{Competing interests}

The authors declare that they have no competing interests.

\section{Publisher's note}

Springer Nature remains neutral with regard to jurisdictional claims in published maps and institutional affiliations.

Received: 21 June 2018 Accepted: 11 September 2018

Published online: 20 September 2018

\section{References}

1. Siegel RL, Miller KD, Jemal A. Cancer statistics, 2016. CA Cancer J Clin. 2016; 66(1):7-30.

2. Gridelli C, Rossi A, Carbone DP, Guarize J, Karachaliou N, Mok T, et al. Non-small-cell lung cancer. Nat Rev Dis Primers. 2015;1:15009.

3. Soda M, Choi YL, Enomoto M, Takada S, Yamashita Y, Ishikawa S, et al. Identification of the transforming EML4-ALK fusion gene in non-small-cell lung cancer. Nature. 2007:448(7153):561-6.

4. Mano H. Non-solid oncogenes in solid tumors: EML4-ALK fusion genes in lung cancer. Cancer Sci. 2008;99(12):2349-55.

5. Horn L, Pao W. EML4-ALK: honing in on a new target in non-small-cell lung cancer. J Clin Oncol. 2009;27(26):4232-5.

6. Rybak-Wolf A, Stottmeister C, Glazar P, Jens M, Pino N, Giusti S, et al. Circular RNAs in the mammalian brain are highly abundant, conserved, and dynamically expressed. Mol Cell. 2015:58(5):870-85.

7. Guarnerio J, Bezzi M, Jeong JC, Paffenholz SV, Berry K, Naldini MM, et al. Oncogenic role of fusion-circRNAs derived from cancer-associated chromosomal translocations. Cell. 2016;165(2):289-302.
8. Tan S, Gou Q, Pu W, Guo C, Yang Y, Wu K, et al. Circular RNA F-circEA produced from EML4-ALK fusion gene as a novel liquid biopsy biomarker for non-small cell lung cancer. Cell Res. 2018;28(6):693-5.

9. Han D, Li J, Wang H, Su X, Hou J, Gu Y, Qian C, et al. Circular RNA MTO1 acts as the sponge of miR-9 to suppress hepatocellular carcinoma progression. Hepatology. 2017;66(4):1151-64.

10. Li Y, Zheng Q, Bao C, Li S, Guo W, Zhao J, et al. Circular RNA is enriched and stable in exosomes: a promising biomarker for cancer diagnosis. Cell Res. 2015:25(8):981-4.

\section{Ready to submit your research? Choose BMC and benefit from:}

- fast, convenient online submission

- thorough peer review by experienced researchers in your field

- rapid publication on acceptance

- support for research data, including large and complex data types

- gold Open Access which fosters wider collaboration and increased citations

- maximum visibility for your research: over $100 \mathrm{M}$ website views per year

At $\mathrm{BMC}$, research is always in progress.

Learn more biomedcentral.com/submissions 